\title{
A hybrid tree sensor network for a condition monitoring system to optimise maintenance policy
}

\author{
Lorenzo Ciani ${ }^{1}$, Alessandro Bartolini ${ }^{1}$, Giulia Guidi ${ }^{1}$, Gabriele Patrizi ${ }^{1}$ \\ ${ }^{1}$ University of Florence, Department of Information Engineering (DINFO), via di S.Marta 3, 50139, Florence, Italy
}

\section{ABSTRACT}

Wind energy is the leading candidate among renewable energy sources as an alternative to burning fossil fuels. A proper and accurate condition monitoring plan is necessary to ensure the high reliability and availability required by energy production systems. This article proposes a wireless mesh network to implement a widely distributed condition monitoring system for a wind farm. Using different types of sensor, the condition monitoring system evaluates the health state of each turbine. The aim of the work is to propose an architecture that can identify possible incipient failures in the most critical turbine's components. Using this system, it is possible to guarantee continuity of service minimising the unplanned maintenance operation due to hidden failure.

\section{Section: RESEARCH PAPER}

Keywords: Wireless Sensor Network; Condition Monitoring; Maintenance; Wind Turbine, Redundancy

Citation: Lorenzo Ciani, Alessandro Bartolini, Giulia Guidi, Gabriele Patrizi, A hybrid tree sensor network for a condition monitoring system to optimise maintenance policy, Acta IMEKO, vol. 9, no. 1, article 2, March 2020, identifier: IMEKO-ACTA-09 (2020)-01-02

Editor: Lorenzo Ciani, University of Florence, Italy

Received October 30, 2019; In final form January 14, 2020; Published March 2020

Copyright: This is an open-access article distributed under the terms of the Creative Commons Attribution 3.0 License, which permits unrestricted use, distribution, and reproduction in any medium, provided the original author and source are credited.

Corresponding author: Gabriele Patrizi, e-mail: gabriele.patrizi@unifi.it

\section{INTRODUCTION}

In recent years, the diagnostic process became fundamental in several different fields of applications. Diagnostics are now an essential part of the performance requirements in Industry 4.0, especially for industrial, energy, and transportation systems [1][7]. Diagnostics are used to evaluate the current health status of the system under test, predicting its remaining useful life based on features that capture the gradual degradation of its operational capabilities. The diagnostic procedure allows the improvement of the Reliability, Availability, Maintainability, and Safety (RAMS) parameters of complex systems, ensuring that the equipment works successfully and reducing downtime due to unexpected failures [8]-[12].

Condition Monitoring (CM) is one of the most effective and powerful diagnostic procedures. $\mathrm{CM}$ is the process of monitoring one or more condition parameters in machinery to identify some changes that are indicative of an incipient fault or equipment health degradation [13]. In the past, CM was applied simply through routine manual diagnostic actions; however, with the introduction of low-cost sensors and automated monitoring systems, online data-driven CM was adopted.

$\mathrm{CM}$ is a type of Condition-Based Maintenance (CBM) used to select and survey parameters from the sensors placed in the system in order to detect a change in the health machine condition [14][15]. CM, as well as reliability and availability, is mandatory in several manufacturing fields, such as energy and industry applications, where products are forced to endure extreme process and environmental conditions [7][16]-[18].

During the design phase of a product or plant, assessment of the optimal diagnostic and failure management policy is a critical phase that could be implemented using the Reliability-Centred Maintenance (RCM) procedure. RCM allows the efficient and effective achievement of the required safety, availability, and economy of operation. RCM provides a decision process to identify applicable and effective diagnostic and preventive maintenance requirements for equipment in accordance with the safety-related, operational, and economic consequences of identifiable failures as well as the degradation mechanisms responsible for those failures. RCM uses a Failure Modes and Effects Analysis (FMEA) to lead the diagnostic and maintenance assessment towards the most cost-effective solution. According to the international standard IEC 60300-3-11, the RCM procedure follows five steps, providing a comprehensive programme that addresses not only the analysis process but also the preliminary and follow-on activities necessary to ensure that the RCM effort achieves the desired results [19]-[22]:
1. Initial definition (availability requirements, maintenance specification, environment, and so on);
2. Failure modes and effects analysis; 
3. Identification of the optimal maintenance task using the decision-making diagram;

4. Implementation of corrective actions; and

5. Continuous improvements that give information to improve steps 2 and 3 .

Steps 2 and 3 are the core of RCM. Indeed, FMEA is a systematic procedure for the analysis of a system to identify the potential failure modes, their causes and effects on system performance. It allows the identification of the criticality of each failure mode, and it provides an input to mitigate the risk associated with the modes [23]. The 'Identification of the Optimal Maintenance Task' (step 3) is guided by the FMEA report, which provides important information for selecting the most appropriate and effective failure management policy. In compliance with international standard IEC 60300-3-11, the main maintenance tasks are [22]:

- $\quad \mathrm{CM}$ : the continuous or periodic task of evaluating the condition of an item in operation against preset parameters in order to monitor its deterioration. It may consist of inspection tasks, which are an examination of an item against a specific standard.

- Scheduled restoration: the work necessary to return the item to a specific standard. Since restoration may vary from cleaning to the replacement of multiple parts, the scope of each assigned restoration task must be specified.

- Scheduled replacement: the removal from service of an item at a specified life limit and the replacement thereof by an item meeting all the required performance standards.

- Failure finding: the task of determining whether or not an item is able to fulfil its intended function. It is solely intended to reveal hidden failures. A failure-finding task may vary from a visual check to a quantitative evaluation against a specific performance standard. Some applications restrict the ability to conduct a complete functional test. In such cases, a partial functional test may be applicable.

- No preventive maintenance: in some situations, no task is required, depending on the effect of failure. The result of this failure management policy is corrective maintenance or no maintenance at all. Sometimes, this approach is called 'run-to-failure'.

$\mathrm{CM}$ and failure finding belong to the CBM procedure. CBM is the most effective policy because its goal is to monitor the health state of the system under test and to start a maintenance intervention only in the case that it is necessary [24]-[27]. In fact, international standard IEC 60300-3-11 proposes a decision diagram for the task assessment that assigns the $\mathrm{CM}$ as the optimal maintenance solution for improving system availability and minimising unexpected failures. For this reason, this article proposes a CM network for a wind farm based on a customised low-cost wireless sensor network.

\section{SYSTEM UNDER TEST}

A wind farm, also known as a wind park, is a group of Wind Turbines (WTs) in the same location used to produce electricity. A large wind farm may consist of several hundred individual W'Ts and may cover an extended area of hundreds of square kilometres, but the land between the turbines may be used for

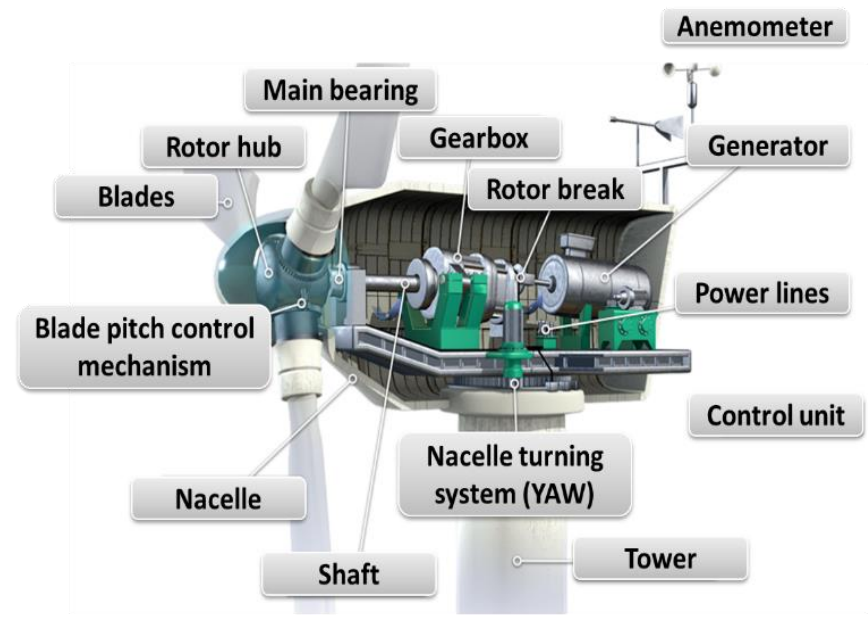

Figure 1. Internal view of a horizontal-axis wind turbine highlighting the most critical components.

agricultural or other purposes [28]-[30]. This results in a very critical issue during the design of CM and diagnostic systems.

A WT is a complex equipment made up of both mechanical and electrical/electronic components. The efficiency and practicality of horizontal-axis three-blade turbines have resulted in domination of the market, and in almost all the new installations of wind farms, this is the only type that is employed for energy generation. Such a wind turbine is comprised of four major components (the rotor, tower, blades, and nacelle) in an enclosure that contains the electrical, electronical, mechanical, and hydraulic components needed to produce electricity (e.g. gearbox, shafts, rotor brake, and nacelle turning system, usually known as yaw, generator, power lines, control system, bearings, pitch controller, and so on). Figure 1 shows the main components of a horizontal-axis, three-blade WT.

This article focuses on a Spanish wind farm located near Zaragoza in the region of Aragon (Spain). The first phase of the project has the aim of analysing all the components included in the turbine and to carry out a FMEA, which highlights several different types of failure modes and causes. Many causes have a great influence on the electronic and mechanical components; for instance, failures of the mechanical items may be affected by high vibration levels on the shafts, the very extreme temperature generated during the rotation speed conversion by the gearbox, corrosion, and fatigue. The damage to the electrical and electronic components may be affected by the environmental conditions, such as temperature, humidity, and vibration.

\section{PROPOSED WIRELESS SENSOR NETWORK TO MONITOR THE WIND FARM UNDER TEST}

Monitoring systems play a fundamental role in any wind farm, because they are crucial to guaranteeing the turbine functionality and to providing quick advice in the case of failure. Furthermore, the recent development of offshore wind farms makes the diagnostic implementation to control and manage the status of the turbines even more difficult. For example, the use of a wired monitoring system would entail higher additional costs compared to the wireless solution. Moreover, in a fixed installation, technological and structural change is more difficult.

Therefore, it is essential to use a low-cost plug-and-play system, such as a Wireless Sensor Network (WSN). WSNs are a network characterised by a distributed architecture of small nodes. Each node can host multiple sensors and is equipped with 
a computational and wireless communication unit [31][32]. The monitoring system acquires several condition parameters to evaluate the health state of the turbine. It must be able to detect and notify potential breakdowns in time, thereby preventing critical damage and increasing the WT's operational lifetime.

However, the design of a monitoring system for a wind farm has four main issues:

- the large dimensions of the covered area;

- the great number of parameters that must be monitored;

- the condition parameters rapidly change, and they therefore require a high sampling frequency;

- the possibility of increasing the number of sensor nodes in the case of new turbine installations inside the wind farm.

The problems explained previously require a proper trade-off in terms of the cover area and transmission protocol. In fact, the great number of parameters and the high sampling frequency require a high-frequency protocol with a high bitrate that usually is characterised by a limited cover area. Wireless Mesh Networks (WMNs) are an optimal solution for providing broadband internet access to large geographical areas using a great number of near nodes and dynamic routing tables to guarantee highfrequency transmissions and a high bitrate, despite the large geographical area [33]. Moreover, WMNs allow the increase of the number of nodes inside the network in the case of a new turbine installation without requiring any change in the already existing architecture, with a consequent decrease of management cost.

The differences between a traditional WSN and a WMN are as follows [34]-[37]:

- A traditional WSN (Figure 2) is a point-tomultipoint (star) network where a single central node, known as the Access Point (AP), is directly connected to all other nodes known as stations or, generally, nodes. Traditional infrastructure Wi-Fi networks have the disadvantage of a limited coverage area because every station must be in a range directly connected with the AP. Furthermore, overloading is another relevant drawback of traditional wireless networks, because the maximum number of stations permitted in the network is limited by the capacity of the AP.

- A WMN (Figure 3) is a self-organised and selfconfigured sensors network. It is composed of several sensor nodes and a single root node that

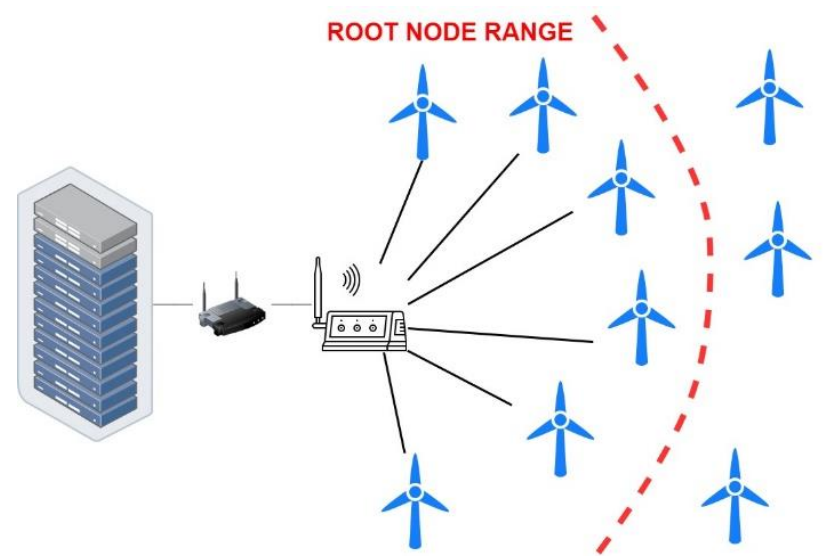

Figure 2. Traditional wireless sensor network.

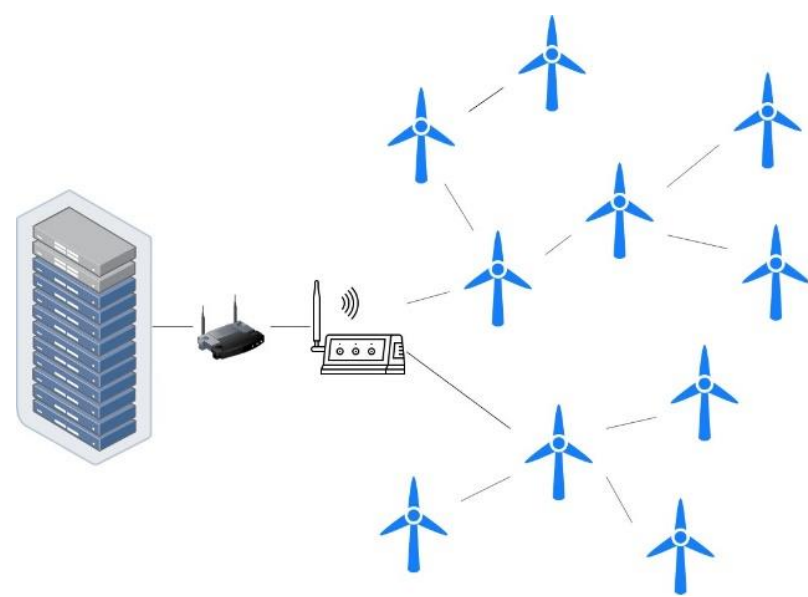

Figure 3. Wireless mesh network topology.

manages the entire network and allows for data uploading to the cloud. Each node can communicate with all the neighbouring nodes. Consequently, the network can cover very large areas. Another advantage of this network is that it is naturally a fault-tolerant system by definition; in fact, when a node stops working, the whole network does not fail, but the AP can be reached by different paths.

This article proposes the design of a WMN composed of lowcost and low-power sensor nodes based on a commercial system on a chip that integrates a high computational capability microcontroller; multiple analogue and digital inputs; and a multi-protocol radio unit. A block diagram of the proposed architecture is shown in Figure 4.

Figure 4 highlights the main components that make up this system, as follows:

- Power unit: It provides power to all units of the sensor node. In particular, it is composed of a charge regulator, two batteries, and a DC/DC converter. The batteries are necessary for guaranteeing a continuous power supply to the monitoring system. Using this architecture, the monitoring system can provide a continuous flow of information regarding the state of the turbine, even in the case of major damage to the turbine's uninterruptible power supply (UPS). Such a redundancy is mandatory for guaranteeing diagnostic self-sufficiency and consequently allowing the proper monitoring of the system under test.

- Processing unit: the ESP32 powered by Espressif is the system on a chip used to process the acquired data. This unit is composed of a microprocessor that elaborates on the data from the sensors and transmits it using the radio unit. There is also a nonvolatile memory for storing the firmware and a faster volatile memory (RAM) for the data elaboration.

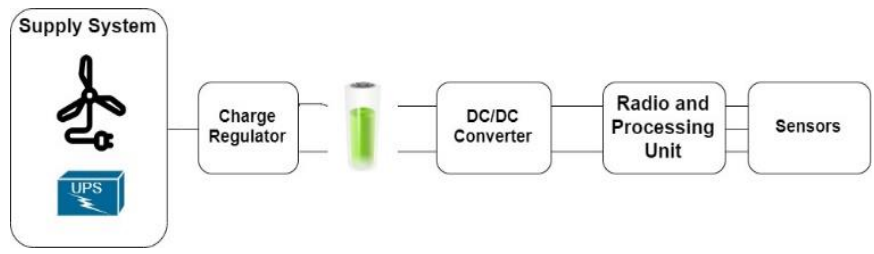

Figure 4. Block diagram of the sensor node. 
- Radio unit: Once the elaboration on the sensor information is complete, the data is transmitted to the central base station using the radio unit. It consists of a RF transceiver and an antenna for transmitting and receiving packets. In order to optimise energy efficiency, the communication unit is set at 'idle and sleep mode'. The ESP32 includes (in the same chip) the processing unit and the radio unit. The protocol used is the IEEE 802.11 (Wi-Fi).

- Sensor unit: it could include several different kinds of transducer, such as thermal, vibration, magnetic sensors, etc., based on the physical quantities under analysis. If one or more sensors provide analogue signals, then they will be converted to digital form using an Analogue-to-Digital Converter (ADC).

The WMNs provide continuous and near real-time data acquisition and autonomous data acquisition. It can be used to [38]:

- maintain process tolerances;

- $\quad$ verify and protect machines, systems, and process stability;

- detect maintenance requirements;

- minimise downtime;

- prevent failures and consequently save costs and time; and

- plan a maintenance policy based on the prediction of failure.

Each turbine requires hundreds of different sensors to monitor the condition parameters that influence the wear-out process; therefore, a hybrid tree-mesh network is proposed. Figure 5 shows the network architecture divided into three layers:

- the first level is composed only of the root node;

- the second layer is the Wi-Fi mesh network where each node identifies a specific turbine; and

- the third layer is a wired network directly connected only to the second layer node of the specific turbine.

The root node is the data collection point of the network and also includes the router and server, as shown in Figure 6. Each of the nodes in the second layer manages the data collection of a single wind turbine. It is also able to work as bridge node for the other second-layer devices receiving data from a different node and retransmitting that to the root node in order to cover the entire wind farm area. Inside each turbine, there are a few thirdlayer nodes with the aim of managing various sensors, as shown in Figure 6 and Figure 7. This level uses a shielded wired connection to carry information to the second-layer node and to avoid interference caused by electronic and mechanical components of the turbine. Figure 7 also slows an example of

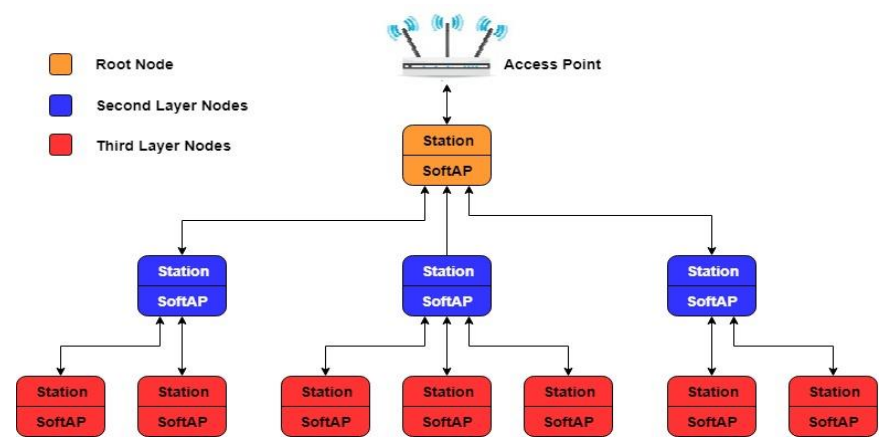

Figure 5. Hybrid tree-mesh network architecture.

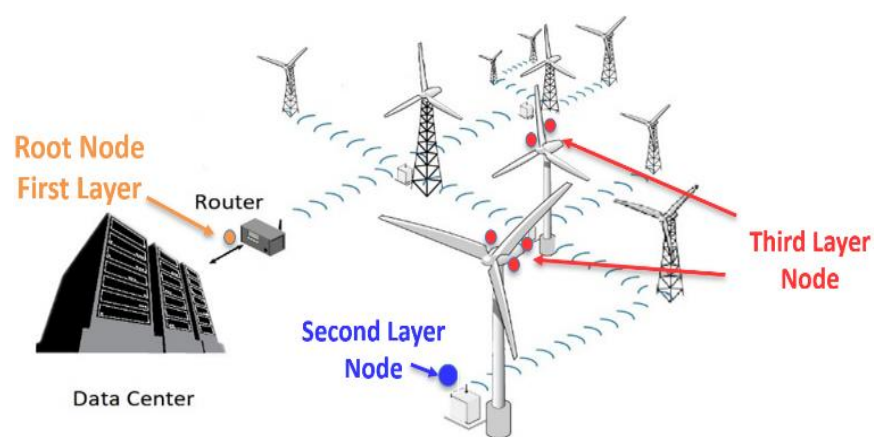

Figure 6. Proposed architecture with first-, second-, and third-layer node location.

diagnostic implementation using the proposed network in the nacelle of the WT under test. The red circle shows some possible locations for the processing unit of the nodes to manage the sensors of a specific turbine section.

Over the years, the power generation capability of the WT has increased, as well as the dimension of the blades and the overall WT extension. Therefore, the distance between two neighbouring WTs could be significant (over hundreds of metres) [39]. The ESP32 board is equipped with an integrated patch antenna, which is definitely not enough to cover such distances, so it is necessary to introduce another antenna located above the nacelle used for the transmission of the acquired data (Figure 7). It is not possible to use a directional antenna heading towards a close node, because the WMN is a self-organised and self-configured network in which every device must be able to communicate with many nodes located in different positions. Therefore, a power amplifier and a high-gain omnidirectional antenna are used as a radio unit. Consequently, the second-layer node is located outside the WT, close to the antenna. It is very important to protect the electronic device by the weathering effects using a protective case to minimise the acceleration of the wear-out process due to temperature, humidity, rain, wind, and solar radiation.

\section{OPTIMIZATION OF MAINTENANCE POLICY}

An RCM procedure was carried out on the wind farm under test to identify the failure mode that could be monitored using condition parameters [40]. Table 1 presents an extract of the RCM assessment, containing:

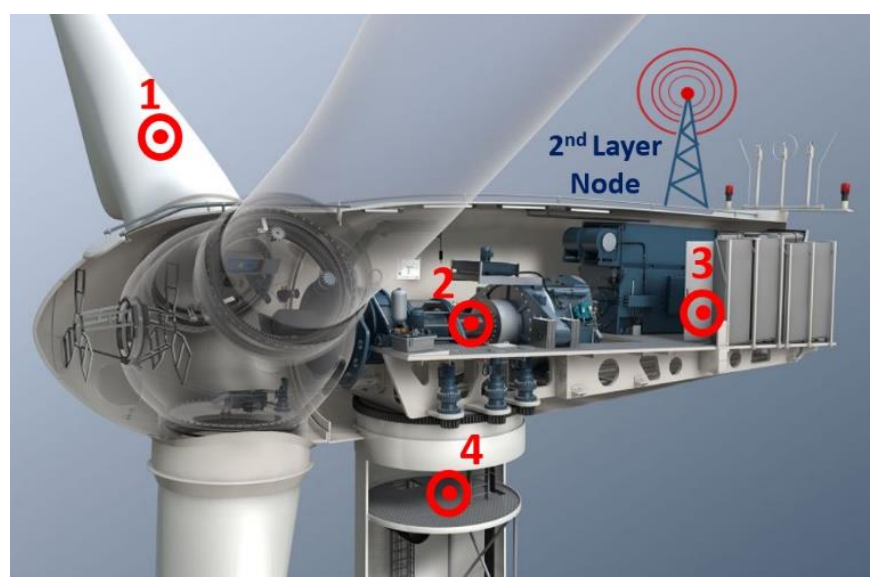

Figure 7. Possible third-level sensor (red circle) deployment inside the nacelle of a wind turbine. 
Table 1. Extract of RCM assessment for the most critical component of the WT

\begin{tabular}{|c|c|c|c|c|}
\hline Component & Description/Function & Failure modes and mechanisms & Failure effects & Maintenance task \\
\hline \multirow{4}{*}{ Blades } & \multirow{4}{*}{$\begin{array}{l}\text { Power source of the wind turbine that } \\
\text { catch the wind energy and convert it in } \\
\text { mechanical motion. }\end{array}$} & - Fatigue failure & \multirow{4}{*}{$\begin{array}{l}\text { Loss of aerodynamics and effiency } \\
\text { property }\end{array}$} & \multirow{4}{*}{ Condition monitoring } \\
\hline & & - Microfracture & & \\
\hline & & - Corrosion & & \\
\hline & & - Delamination & & \\
\hline \multirow{4}{*}{ Gearbox } & \multirow{4}{*}{$\begin{array}{l}\text { Increases the rotor speed up to the } \\
\text { appropriate generator speed using gears, } \\
\text { pinions and shafts. }\end{array}$} & - Binding/Sticking & \multirow{4}{*}{ Inoperative WT } & \multirow{4}{*}{$\begin{array}{l}\text { Condition monitoring combined with } \\
\text { failure finding and predetermined } \\
\text { maintenance restoration every } 12 \\
\text { months }\end{array}$} \\
\hline & & - Excessive Wear & & \\
\hline & & - Fails to move & & \\
\hline & & - Mechanical damage & & \\
\hline \multirow{3}{*}{ Brake } & \multirow{3}{*}{ Stop the turbine movement on demand } & - Excessive Wear & \multirow{3}{*}{$\begin{array}{l}\text { Impossibility to brake the nacelle } \\
\text { movement }\end{array}$} & \multirow{3}{*}{ Scheduled maintenance every 6 months } \\
\hline & & - Leaking & & \\
\hline & & - Cracked housing & & \\
\hline \multirow{4}{*}{ Hydraulics system } & \multirow{4}{*}{$\begin{array}{l}\text { Moves the nacelle towards the yaw axis } \\
\text { (wind direction) }\end{array}$} & - Leakage & \multirow{4}{*}{$\begin{array}{l}\text { Loss of pressure in the hydraulic } \\
\text { system } \\
\text { Impossible to move the nacelle } \\
\text { toward the wind direction }\end{array}$} & \multirow{4}{*}{$\begin{array}{l}\text { Condition monitoring combined with } \\
\text { predetermined maintenance } \\
\text { restoration every } 6 \text { months }\end{array}$} \\
\hline & & - Contamination & & \\
\hline & & - Improper flow & & \\
\hline & & - Stuck valve & & \\
\hline \multirow{3}{*}{ Tower } & \multirow{3}{*}{$\begin{array}{l}\text { Supports the other parts and holds them } \\
\text { off the ground. }\end{array}$} & - Fatigue failure & \multirow{3}{*}{$\begin{array}{l}\text { Serious damage of the structure. } \\
\text { Possible turbine collapse }\end{array}$} & \multirow{3}{*}{ Condition monitoring } \\
\hline & & - Corrosion & & \\
\hline & & - Fracture & & \\
\hline \multirow{3}{*}{ Bearings } & \multirow{3}{*}{$\begin{array}{l}\text { Achieve a smooth, low-friction rotary } \\
\text { motion or sliding action (linear motion) } \\
\text { between two surface }\end{array}$} & - Binding/Sticking & \multirow{3}{*}{$\begin{array}{l}\text { Possible mechanichal damage } \\
\text { Overheating of the nacelle } \\
\text { Limited turbine functionality }\end{array}$} & \multirow{3}{*}{ Scheduled maintenance every 6 months } \\
\hline & & - Excessive Play & & \\
\hline & & - Contaminated & & \\
\hline \multirow{4}{*}{ UPS } & \multirow{4}{*}{$\begin{array}{l}\text { Provides emergency power when the } \\
\text { input power source or mains power fails. }\end{array}$} & - Dead batteries cells & \multirow{3}{*}{$\begin{array}{l}\text { No impact in the short term if } \\
\text { standard power supply is working, } \\
\text { otherwise inoperative WT }\end{array}$} & \multirow{4}{*}{$\begin{array}{l}\text { Condition Monitoring combined with } \\
\text { Failure finding (only for cooling fan) }\end{array}$} \\
\hline & & - No output & & \\
\hline & & - Cooling fan can not move & & \\
\hline & & & Overheating of the nacelle & \\
\hline \multirow{3}{*}{ Power converter } & \multirow{3}{*}{$\begin{array}{l}\text { Convert electrical energy from the grid to } \\
\text { supply the control system }\end{array}$} & - No output & No impact in the short term if UPS is & \\
\hline & & - Short circuit & working, otherwise inoperative WT & predetermined maintenance \\
\hline & & - Parameters drift & Loss of power converter capability & \\
\hline & & - Input open & & \\
\hline & Contains all the electrical components in & - No output & $\begin{array}{l}\text { Wrong output data elaborated by } \\
\text { the control system }\end{array}$ & \\
\hline Control system & the turbine, such as power converter, PFC & - Short/Open circuit & & Condition monitoring \\
\hline & & - Error in data storage & Inoperative WT & \\
\hline
\end{tabular}

- a description of the functionality of the items useful for a functional failure analysis;

- a list of the main failure modes (how we can observe the inability of the item to perform a required function) and mechanisms (the physical, chemical, or other processes that have led to a failure);

- $\quad$ one or more failure effects related to each failure mode;

- the output of the procedure (the assessed maintenance task) evaluated following the guidelines of international standard IEC 60300-3-11 [22].

The table highlights that $\mathrm{CM}$ is a fundamental management policy for the WT under test, and almost all the subsystems of the turbine can be monitored using the appropriate sensors.

Table 2 deals only with the components for which RCM suggests $\mathrm{CM}$ as the optimal maintenance task in terms of availability, management cost, safety, and downtime. The table shows the types of measurements used to monitor each item and the proposed sensor technology for the relative measurement. The WMN proposed in the previous paragraph is used to implement the CM procedure, and each sensor node is provided by several different types of sensing elements. An IEEE $802.11 \mathrm{n}$ LAN protocol was implemented in the sensor network because it represents the optimal trade-off between the geographical coverage, the transmission of great amount of data, and the power dissipation [41].

\section{RELIABILITY IMPROVEMENTS USING REDUNDANCY}

The WMN proposed in this paper is a diagnostic tool fundamental for the wind farm under test. The entire network is composed of several components forced to endure extreme conditions. Usually, during the reliability analysis of a system or plant, diagnostics involve high-quality components and are considered failure-free. This article also considers the reliability of the diagnostic network to highlight the possible criticality of the proposed configuration.

The mesh network is a fault tolerant system by definition. In fact, when a node fails, the other nodes reconfigure the network to find another path to reach the root node and therefore the server. In this kind of architecture, it is clear that the most critical component becomes the root node because a failure in the root node causes the failure of the whole network. To maximise network reliability and reduce the downtime of the CM system of the mesh network, a warm standby redundancy was considered. This configuration is composed of two root nodes and a switch that detects the state of the main root node and reconfigures the architecture, activating the standby module to maintain the network's operation in case of failure [42][43] (Figure 8).

Both root nodes are connected to power sources, but only the main power source is used. The standby is half-operative and ready to work in the case that the main power source fails. For 
Table 2. Extract of the sensor used to monitor the most critical item.

\begin{tabular}{lll}
\hline Component & Measurement & Sensors \\
\hline Blades & Crack/Fatigue detection & Acoustic Emission \\
& Wind speed/direction & Anemometer \\
& Acceleration, pitch, roll and yaw & Inertial module \\
& Microdeformation & Extensometer \\
\hline Gearbox & Vibration & MEMS accelerometer \\
& Temperature & Thermocouple \\
& Motion & Displacement transducer \\
& Stress & Strain gauge \\
\hline Hydraulics & Pressure & Piezoelectric pressure transmitter \\
system & Flow & Ultrasonic meter \\
& Level & Magnetic level meter \\
& Contamination & Contamination sensor \\
\hline Tower & Crack/Fatigue detection & Acoustic Emission \\
& Microdeformation & Extensometer \\
\hline UPS & Temperature & Resistance temperature detector \\
& Humidity & Humidity sensor \\
& Current & Hall effect integrated current sensor \\
\hline Power & Temperature & Resistance temperature detector \\
converter & Humidity & Humidity sensor \\
& Vibration & MEMS accelerometer \\
\hline Control & Temperature & Resistance temperature detector \\
system & Humidity & Humidity sensor \\
& Vibration & MEMS accelerometer \\
\hline & & \\
\hline
\end{tabular}

this reason, it is not necessary to wait for the standby unit startup because the equipment is ready to be used [43]. The failure rate of the two different configurations was evaluated using a reliability prediction procedure, and the reliability results are shown in Figure 9. The reliability of the root node remains very high, even after several years of the network life cycle because of the limited number of devices that make up the board. The difference between the single item and the warm standby redundancy reliability trends is quite remarkable. In particular, the warm standby allows the enhancement of the network's life cycle, maintaining the reliability as very close to one.

\section{CONCLUSIONS}

Wireless sensor network and condition monitoring represent the optimal maintenance solution for an extended wind farm. This is because of the limited installation and management costs and the possibility of easy installation and/or future upgrade, even if the park is already in use. The article focused on the reliability-centred maintenance procedure, which leads to the optimal maintenance task choice. The first part of the article described the advantages of the proposed hybrid tree wireless mesh network and illustrated its possible deployment for the sensor nodes inside a wind farm. An extract of the reliabilitycentred maintenance procedure for the wind turbine is proposed, and Table 1 shows that for most of the failure modes, the optimal maintenance task is condition monitoring. Indeed, the use of condition monitoring allows the minimisation of scheduled maintenance and consequently saves time and money. Using this approach, maintenance and tasks policies are managed by the diagnostic mesh network. Corrective maintenance is performed

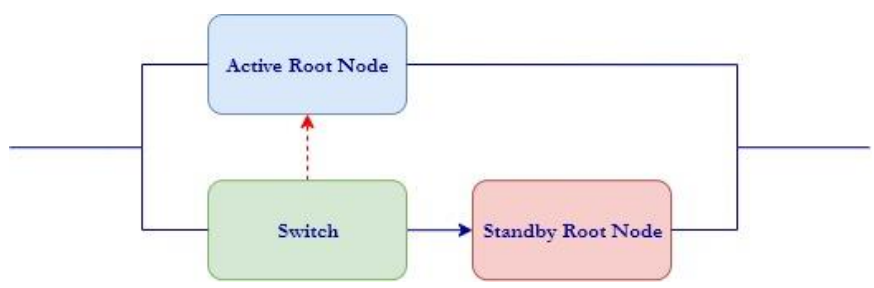

Figure 8. Warm standby architecture proposed for the root node.

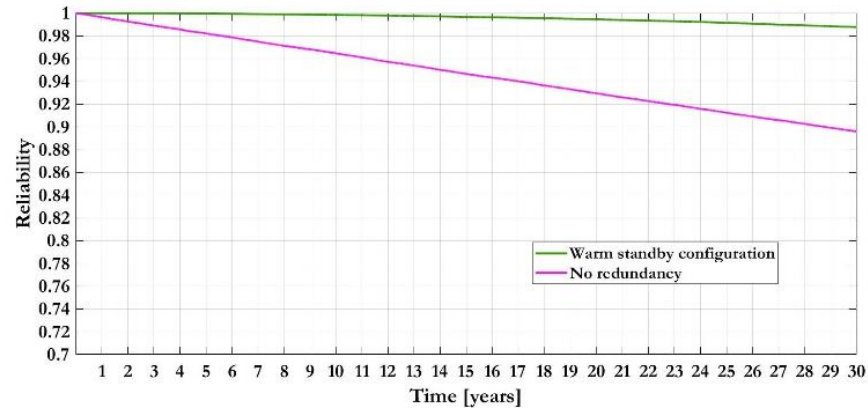

Figure 9. Comparison of the predicted reliability trend without redundancy and using a warm standby configuration.

only in the case that the system identifies an intervention request. The article suggested a group of measurement quantities and relative appropriate sensors to monitor the failure modes identified through the reliability-centred maintenance procedure.

Due to the limited costs of the proposed elaboration unit, the introduction of a fault-tolerant design guarantees a constant service over time without affecting the overall cost. So, finally, a warm standby architecture was designed for the root node, and reliability with and without redundancy is compared in order to show the high increase of the reliability with the warm standby architecture. The root node has to manage all the network functionalities; therefore, the proposed warm standby configuration activates the redundancy in the case of failure, guaranteeing a probability of failure of the root node lower than $0.01 \%$.

\section{REFERENCES}

[1] D. Capriglione, M. Carratu, A. Pietrosanto, P. Sommella, Online Fault Detection of Rear Stroke Suspension Sensor in Motorcycle, IEEE Trans. Instrum. Meas. 68(5) (2019) pp. 1362-1372.

[2] G. Kohlrusz, K. Enisz, B. Csomós, D. Fodor, Electric Energy Converter Development and Diagnostics in Mixed-Signal Simulation Environment, ACTA IMEKO 7(1) (2018) p. 20.

[3] I. Bodini, M. Lancini, S. Pasinetti, D. Vetturi, Techniques for OnBoard Vibrational Passenger Comfort Monitoring in Public Transport, ACTA IMEKO 3(4) (2014) p. 32.

[4] D. Capriglione, M. Carratù, C. Liguori, V. Paciello, P. Sommella, A Soft Stroke Sensor for Motorcycle Rear Suspension, Measurement 106 (2017) pp. 46-52.

[5] D. Capriglione, M. Carratù, A. Pietrosanto, P. Sommella, NARX ANN-Based Instrument Fault Detection in Motorcycle, Measurement 117 (2018) pp. 304-311.

[6] A. Reatti, M. K. Kazimierczuk, M. Catelani, L. Ciani, Monitoring and Field Data Acquisition System for Hybrid Static Concentrator Plant, Measurement 98 (2017) pp. 384-392.

[7] P. Gangsar, R. Tiwari, A Support Vector Machine Based Fault Diagnostics of Induction Motors for Practical Situation of MultiSensor Limited Data Case, Measurement 135 (2019) pp. 694-711.

[8] V. de Vita, G. D. L. di Leo, G. Fabbrocini, C. Liguori, A. Paolillo, P. Sommella, Statistical Techniques Applied to the Automatic Diagnosis of Dermoscopic Images, ACTA IMEKO 1(1) (2012) p. 7.

[9] E. Petritoli, F. Leccese, M. Botticelli, S. Pizzuti, F. Pieroni, A RAMS Analysis for a Precision Scale-Up Configuration of 'Smart Street' Pilot Site: An Industry 4.0 Case Study, ACTA IMEKO 8(2) (2019) p. 3.

[10] A. Ayachit, A. Reatti, M. K. Kazimierczuk, Small-Signal Modeling of PWM Dual-SEPIC DC-DC Converter by Circuit Averaging Technique, Proc. of the 42nd Annual Conference of the IEEE Industrial Electronics Society, IECON 2016, 2016, pp. 3606-3611.

[11] F. Corti, F. Grasso, A. Reatti, A. Ayachit, D. K. Saini, M. K. Kazimierczuk, Design of Class-E ZVS Inverter with Loosely- 
Coupled Transformer at Fixed Coupling Coefficient, Proc. of the 42nd Annual Conference of the IEEE Industrial Electronics Society, IECON 2016, 2016, pp. 5627-5632.

[12] MIL-HDB-338B, Electronic Reliability Design Handbook, US Department of Defense, Washington DC, 1998.

[13] Y. Wang, Z. Zhu, H. Song, K. Shi, Wind Turbine Gearbox Condition Monitoring Based on Extreme Gradient Boosting, Proc. of the 43rd Annual Conference of the IEEE Industrial Electronics Society, IECON 2017, 2017, pp. 6017-6023.

[14] K. Singh, H. Malik, R. Sharma, Condition Monitoring of Wind Turbine Gearbox Using Electrical Signatures, Proc. of the International Conference on Microelectronic Devices, Circuits and Systems, ICMDCS 2017, 2017, pp. 1-6.

[15] L. Albertoni, F. Grasso, J. Matteucci, M. C. Piccirilli, A. Reatti, A. Ayachit, M. K. Kazimierczuk, Analysis and Design of Full-Bridge Class-DE Inverter at Fixed Duty Cycle, Proc. of the 42nd Annual Conference of the IEEE Industrial Electronics Society, IECON 2016, 2016, pp. 5609-5614.

[16] G. Fontana, F. Grasso, A. Luchetta, S. Manetti, M. C. Piccirilli, A. Reatti, A New Simulation Program for Analog Circuits Using Symbolic Analysis Techniques, Proc. of the 2015 International Conference on Synthesis, Modeling, Analysis and Simulation Methods and Applications to Circuit Design, SMACD 2015, 2015, pp. 1-4.

[17] A. Reatti, M. Balzani, PWM Switch Model of a Buck-Boost Converter Operated under Discontinuous Conduction Mode, Proc. of the 48th Midwest Symposium on Circuits and Systems, 2005, pp. 667-670.

[18] J. Moubray, Reliability Centered Maintenance (2nd ed.) Butterworth-Heinemann, 1997.

[19] N. B. Bloom, Reliability Centered Maintenance: Implementation Made Simple, McGraw-Hill Companies, 2006.

[20] NASA Headquarters, Reliability Centered Maintenance Guide for Facilities and Collateral Equipment, 2000.

[21] IEC 60300-3-11, Dependability Management - Part 3-11 Application Guide - Reliability Centred Maintenance, International Electrotechnical Commission, 2009.

[22] L. Cristaldi, M. Khalil, P. Soulatintork, A Root Cause Analysis and a Risk Evaluation of PV Balance of System Failures, ACTA IMEKO 6(4) (2017) p. 113

[23] M. C. Magro, P. Pinceti, M. Antonelli, E. de Paola, P. Firpo, E. Marino, CBM for a Fleet of Railway Vehicles: Infrastructure and Algorithms, ACTA IMEKO 5(4) (2016) pp. 56-63.

[24] D. Seneviratne, L. Ciani, M. Catelani, D. Galar, Smart Maintenance and Inspection of Linear Assets: An Industry 4.0 Approach, ACTA IMEKO 7(1) (2018) p. 50.

[25] E. de Francesco, R. de Francesco, F. Leccese, M. Cagnetti, A Proposal to Update LSA Databases for an Operational Availability Based on Autonomic Logistic, Proc. of IEEE Metrology for Aerospace (MetroAeroSpace), 2015, pp. 38-43.

[26] E. de Francesco, R. de Francesco, F. Leccese, M. Cagnetti, A Proposal to Improve the System Life Cycle Support of Composites Structures Mapping Zonal Testing Data on LSA Databases, Proc. of IEEE Metrology for Aerospace (MetroAeroSpace), 2016, pp. 151-155.

[27] A. Hemami, Wind Turbine Technology, Cengage Learning, 2012.
[28] T. Burton, D. Sharpe, N. Jenkins, E. Bossanyi, Wind Energy Handbook, John Wiley \& Sons, 2001.

[29] M. Caciotta, V. Cerqua, F. Leccese, S. Giarnetti, Ettore De Francesco, Eduardo De Francesco, N. Scaldarella, A First Study on Prognostic System for Electric Engines Based on Envelope Analysis, Proc. of IEEE Metrology for Aerospace (MetroAeroSpace), 2014, pp. 362-366.

[30] Y. Huang, C. Gühmann, Wireless Sensor Network for Temperatures Estimation in an Asynchronous Machine Using a Kalman Filter, ACTA IMEKO 7(1) (2018) p. 5.

[31] L. d'Alvia, E. Palermo, S. Rossi, Z. del Prete, Validation of a LowCost Wireless Sensors Node for Museum Environmental Monitoring, ACTA IMEKO 6(3) (2017) p. 45.

[32] K. C. Karthika, Wireless Mesh Network: A Survey, Proc. of the International Conference on Wireless Communications, Signal Processing and Networking, WiSPNET 2016, pp. 1966-1970.

[33] A. Hać, Wireless Sensor Network Designs, Chichester, UK: John Wiley \& Sons, Ltd, 2003.

[34] Y. Zhang, L. Jijun, H. Hu, Wireless Mesh Networking: Architectures, Protocols and Standards, Tailor \& Francis Group, 2007.

[35] V. Pasquali, G. d'Alessandro, R. Gualtieri, F. Leccese, A New Data Logger Based on Raspberry-Pi for Arctic Notostraca Locomotion Investigations, Measurement 110 (2017) pp. 249-256.

[36] V. Pasquali, R. Gualtieri, G. D’Alessandro, M. Granberg, D. Hazlerigg, M. Cagnetti, F. Leccese, Monitoring and Analyzing of Circadian and Ultradian Locomotor Activity Based on RaspberryPi, Electronics 5(4) (2016) p. 58.

[37] V. J. Hodge, S. O'Keefe, M. Weeks, A. Moulds, Wireless Sensor Networks for Condition Monitoring in the Railway Industry: A Survey, IEEE Trans. Intell. Transp. Syst. 16(3) (2015) pp. 10881106

[38] A. González-González, A. Jimenez Cortadi, D. Galar, L. Ciani, Condition Monitoring of Wind Turbine Pitch Controller: A Maintenance Approach, Measurement 123 (2018) pp. 80-93.

[39] L. Ciani, A. Bartolini, G. Guidi, G. Patrizi, Condition Monitoring of Wind Farm based on Wireless Mesh Network, Proc. of the 16th IMEKO TC10 Conference: 'Testing, Diagnostics \& Inspection as a comprehensive value chain for Quality \& Safety', 2019, pp. 3944.

[40] J. Jansons, T. Dorins, Analyzing IEEE 802.11n Standard: Outdoor Performanace, Proc. of the 2012 Second International Conference on Digital Information Processing and Communications, ICDIPC 2012, pp. 26-30.

[41] M. Blanke, M. Kinnaert, J. Lunze, M. Staroswiecki, Diagnosis and Fault-Tolerant Control, Springer Berlin Heidelberg, Berlin, Heidelberg, 2016.

[42] M. Catelani, L. Ciani, A. Bartolini, G. Guidi, G. Patrizi, Standby Redundancy for Reliability Improvement of Wireless Sensor Network, Proc. of the 5th International Forum on Research and Technologies for Society and Industry, 2019.

[43] O. Tannous, L. Xing, P. Rui, M. Xie, S. H. Ng, Redundancy Allocation for Series-Parallel Warm-Standby Systems, Proc. of the 2011 IEEE International Conference on Industrial Engineering and Engineering Management, 2011, pp. 1261-1265. 